\title{
The USE OF RFID TEChNology IN HoSPITAL
}

\author{
Barbora Kotkova \& Martin Hromada
}
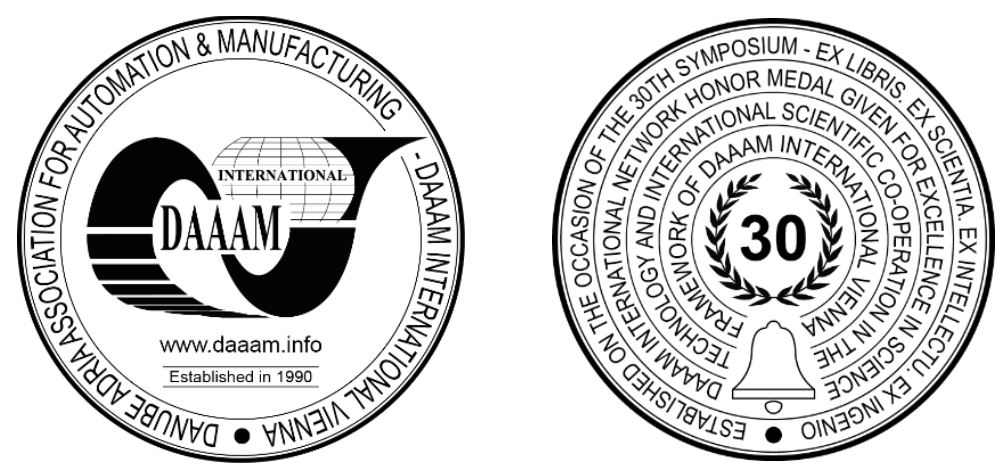

This Publication has to be referred as: Barbora, K[otkova] \& Hromada, M[artin] (2020). The use of RFID Technology in Hospital, Proceedings of the 31st DAAAM International Symposium, pp.0638-0643, B. Katalinic (Ed.), Published by DAAAM International, ISBN 978-3-902734-29-7, ISSN 1726-9679, Vienna, Austria

DOI: $10.2507 / 31$ st.daaam.proceedings.088

\begin{abstract}
The article describes the increase of safety and unambiguous identification of patients during treatment using RFID technology. It discusses the principle on which RFID works, software and hardware requirements, and a comparison of the quality and quantity of data obtained. The reduction of risks in the provision of health care is achieved mainly through preventive measures and early detection of threats. One tool to minimize this adverse event may be to reliably identify the patient, record diagnoses, procedures, and medications that are being administered. Research into the use of RFID is currently underway in several medical centers around the world. Nursing practice is constantly changing, new procedures and drugs are being developed. At the same time, however, this increases the demands on the medical staff, their expertise, and responsibility for the nursing care provided. And it is here, where a detailed and error-free overview of patient identification is required (especially in a situation where the identity cannot be verified by questioning), the procedures he has undergone or is to undergo, and prescribed drugs, RFID technology is a reliable helper.
\end{abstract}

Keywords: identification; information; technology; veryfication.

\section{Radio Frequency Identification-RFID}

RFID - Radio Frequency IDentification is one of the most modern technologies and is used to identify objects using radiofrequency waves. It is currently beginning to replace bar code identification technology. In contrast to bar code technology (which works with only one input and immutable data), radio frequency identification systems can record, store, and provide information in real-time. Therefore, comparisons in the content of this article are very important, what are the benefits of exchanging bar code technology for RFID technology. It summarizes the amount of necessary information that would be obtained by exchanging technologies not only in a very short time, but also quality and quantity.

The beginnings of the development of RFID technology date back to 1886, when Frederick Hertz used various experiments to find that radio waves can bounce off certain objects. Furthermore, in 1935, the Scottish electrician Sir Robert Alexander Watson-Watt invented and designed the first functional device that worked on the principle of microwaves - radar. This was used especially during World War II when Allied aircraft were equipped with radars that we're able to receive the signal sent from the radar station. He immediately sent back to the radar station, which recognized the friendly aircraft from the enemy. The development of radio communication systems continued after World War II from 1950 to 1960. Various types of applications were developed and tested for use in various companies and industries. The first applications were on single-bit chips, the so-called EAS system, the principle of which is still used today. Most often in stores, such as security systems against theft. 
Mario W. Cardullo is considered a pioneer of modern RFID technology, who on January 23, 1973, patented the first active RFID tag with rewritable 16-bit memory, which is still used in the modification. That same year, Charles Walton patented the first passive tag that could unlock a door without a key. The tag placed in the card was attached to the door reader and after recognizing a valid identification number, the door was unlocked. At this stage of development, the US government has already begun to take an interest in RFID systems. At the Los Alamos National Laboratory, the scientists then developed a system to monitor the movement of nuclear waste and material. In the 1990s, IBM patented RFID systems operating in the UHF band, where this frequency range offered a significantly longer reading range and faster data transmission. In connection with the achieved successes, research laboratories were built in Australia, Great Britain, Switzerland, Japan, and China. From these laboratories, RFID technologies have already led the way to all industries. They began to be used as identification methods, for example in automated transport payment systems, identification in tracking goods in shops, and transport around the world. [1]

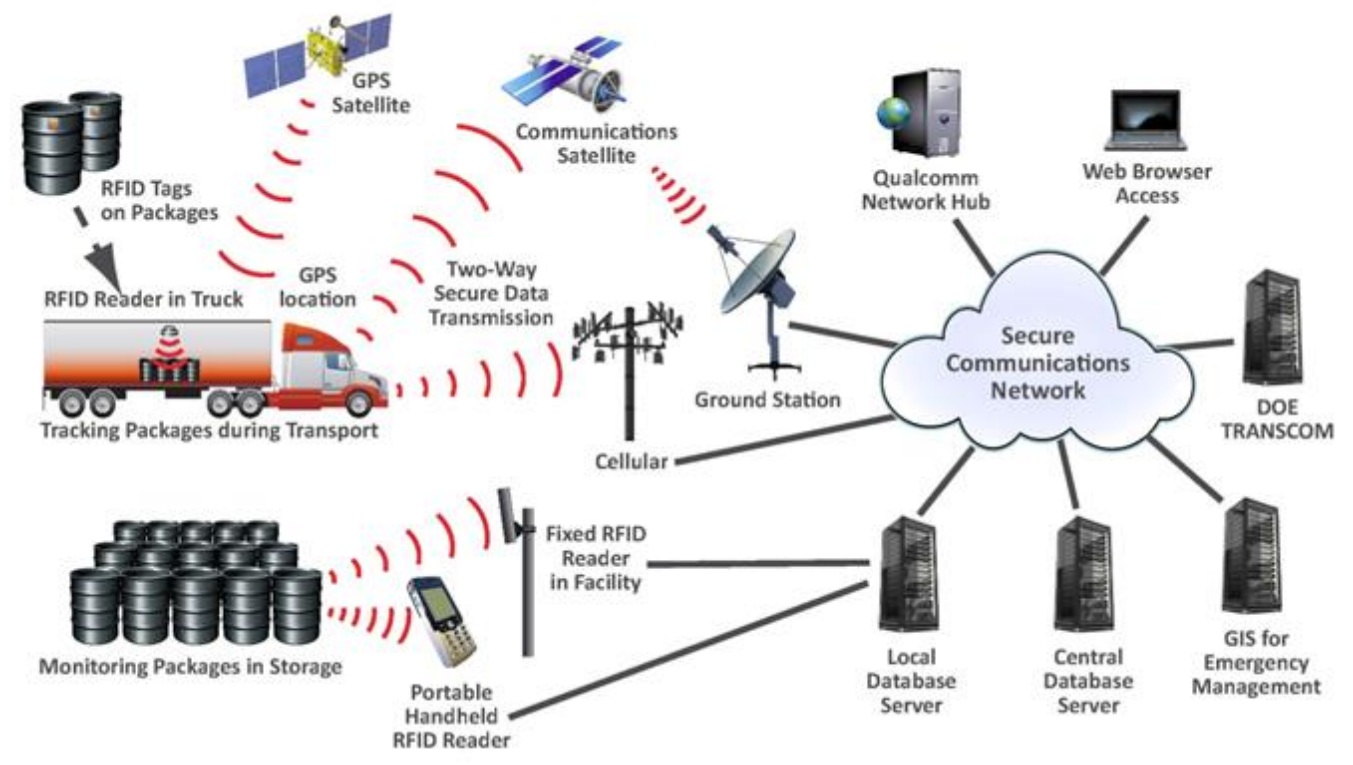

Fig. 1. Figure example [2]

However, in no other sector is such identification accuracy required to eliminate errors than in healthcare. Human health, sometimes life, is at stake. Patient safety represents the reduction of the risk of unnecessary harm associated with the provision of healthcare to an acceptable minimum. Concerns about patient safety are currently rising, increasing in proportion to the rates of errors and injuries resulting from adverse event reports. [3] It is generally reported that 8-12\% of hospitalized patients have experienced healthcare side effects. [4]

To improve safety, healthcare professionals must be able to identify individual patients, which can be particularly problematic when they are unconscious or unable to communicate. The goal of patient safety is therefore to identify him and obtain basic information - who he is, what his diagnosis is, what examinations he has undergone and what he is waiting for, what medicines are prescribed to him, and other necessary information. And it is here that the use of RFID technology is offered, which not only can capture and provide all this information, but it is also possible to update, supplement, and change it. Not only in cases where an event threatening human health or life occurs, but the immediate availability of this information will also be essential to speed up and streamline the work of physicians.

\subsection{Basic components of the RFID system}

The basic components for data storage and transmission include an RFID chip, EPC code, RFID reader, and software.

- The RFID chip (tag) is connected to the antenna, with which it communicates with the RFID reader. RFID chips are divided into passive, active. Passive RFID chips are powered by the energy of transmitted radio waves, they can have an extended memory with the ability to write and read other information. Active RFID chips have their power supply and transmit their identification. Their disadvantage is the higher price and limited service life. RFID chips are available in reading or read and write variants. The operating frequency of the chip is also important, the higher it is, the greater the reading distance. [5]

- The RFID reader is its sensor with an antenna, providing communication with chips and which reads the stored EPC code. RFID readers connect the RFID tag and control the computer. It supplies passive tags with the necessary energy, reads and writes the information stored in the tag, and also transfers it to the control computer. RFID readers can be divided into two basic groups, stationary and mobile readers. [6]

- The EPC code (Electronic Product Code) uniquely identifies each RFID chip. This is a 96-bit unique number.

- Software (middleware) translates and filters data for use in a given information system. [7] 


\subsection{Performance of RFID systems}

The performance of RFID systems can be compromised for several reasons. The most common is their susceptibility to interference from other radio systems, especially those operating at low frequencies. Furthermore, they are adversely affected by the presence of liquids or wet surfaces. Metal is also an electromagnetic reflector through which radio signals cannot penetrate. It can either completely prevent the signal from penetrating or cause unwanted reflections. Improper antenna placement can also cause reading problems, and multiple chips can be read multiple times by multiple cameras. Therefore, it is necessary to pay attention to the correct installation of readers, good design of the RFID network infrastructure, and the way of placing RFID tags. The basic criteria influencing the performance of the system, therefore, include the occurrence of problematic materials, incorrectly chosen frequency band, inappropriate placement of RFID system components, interference of other devices in the same frequency band, and misunderstanding of the RFID system principle.

\subsection{Comparison of RFID and barcode}

When comparing RFID tags and barcodes, it is clear that each of the technologies has its advantages and disadvantages. Compared to barcodes, RFID tags have several major advantages:

- The RFID tag may not be directly visible to the RFID reader and therefore the reading devices don't need to be exposed to mechanical or weather damage. Chips can also be placed inside the product, so they are also not exposed to external influences. Therefore, RFID chips are several times more resistant to barcode tags, which are very prone to damage from scratches, tears, or dirt. [8]

- Multiple readers can read a large number of RFID chips over long distances. The reading distance in the case of RFID tags can be up to 15 meters. Also, the barcodes must be read separately.

- For RFID chips, it is possible to write or change information directly into the RFID tag on the product, thus ensuring clarity about the entire "life cycle" of the product. In contrast, for a barcode, the information is given and a new label must be provided for any change.

- The disadvantages of RFID technology are higher production costs and the price of RFID chips compared to barcodes. [9]

\section{RFID options in medical facilities}

Currently, RFID technology is used in healthcare to identify patients and record their treatment procedures. Initially, the patient is assigned a bracelet with a built-in RFID tag upon admission. Here and in the information system, the medical procedures and procedures that the patient underwent are subsequently recorded. Also, the medication prescribed to him, including the exact dosage. Furthermore, the chip can also be used to monitor the position of patients within a medical facility. RFID can be in the form of a bracelet or implanted under the skin. In addition to identifying and monitoring the position of patients, the technology can also be used to monitor the records and position of objects. Patient location information can then be derived from object location information used by the patient. The collected data is stored in the hospital information system. In this way, they can be transferred between different devices, for example when a patient passes from one ward to another, or when a patient is examined by another medical device. This automated data transfer speeds up the availability of relevant patient information and significantly reduces the risk of human error. Furthermore, in the case of advanced systems, the extracted necessary data can also be sent to suppliers of hospital supplies.

It follows from the above that the possibilities of using RFID technology in medical facilities are relatively wide. Therefore, one department will be specifically described in this article, namely the donor part of the blood center. It consists of the pre-collection phases, the doctor's examination room, the pre-box, and the blood or plasma collection itself. Important parts of the sampling process will be described in the form when using barcodes. It is, therefore, possible to identify a specific blood product, but not important information such as temperature, humidity, who, when, where, and for how long the product was handled. Therefore, subsequently, for an accurate overview of the handling, production, storage, and distribution of blood and blood products will be designed to use RFID chips.

\section{Blood collection and registration using barcodes}

For the identification and registration of blood cans using barcodes, the barcodes themselves, scanners, and computer software are necessary to identify people, where each worker must identify himself with his own card. The whole process has the following main parts: a control sample, a control examination performed by a doctor and it ends with the collection of blood or plasma into a blood can. This is used to transport blood. The designation of the blood can is as follows - a label containing data such as product, collection-number, donor identification number, blood group and Rh factor, date of collection, expiration date, transfusion amount, preservative solution content and composition, storage conditions, serial number, batch number, serial number and manufacturer's designation. The first step is control sampling: the donor must be the first to complete a control questionnaire and is assigned a series of barcodes on the records, used to identify only this donor. Then his temperature and pressure are measured, which are then recorded on his card. 
Subsequently, the donor waits in the waiting room, when it is his turn according to the numerical queue, he moves to the hematology laboratory. A control blood sample is taken here. The same bar code as that issued during the donor's registration is affixed to the test tube with the sample taken. The donor card is then transferred to the doctor's examination room.

The second step is a follow-up examination by a doctor - in the infirmary, the doctor checks the donor's card and questionnaire, and on the computer the results of the control samples. It also determines the amount of blood or plasma to be collected. In the waiting room, he then waits for a call for the collection itself, if everything is in order. The donor goes from the waiting room to pick up, he has the same bar codes remaining in the card. The card is handed over to the nurse, who is preparing blood bags here. They stick the barcodes from the card on this and also on the tube. The computer lists the donor card and the batch number of the bag. The donor will then receive a final questionnaire, which must again be marked with a bar code.

The third step is blood collection. During the collection itself, the nurse must check the donor card again, the data entered into the PC, the bar code on the bag. He will personally ask the donor to take delivery, his number will only be checked visually with the number given in the PC. For the collection itself, the tubes must be marked with a bar code, then the blood is collected in a blood bag. Then the data on the blood bag is entered into the PC (collection time and weight - the type of collection and the code of the collection number must be entered here). After the end of the collection, the bar codes of both the operator, the nurse, and the codes are read from the bag. After sampling, the following data are stored in the computer again: sampling length, quantity, weight number, employee number, and sampling number.

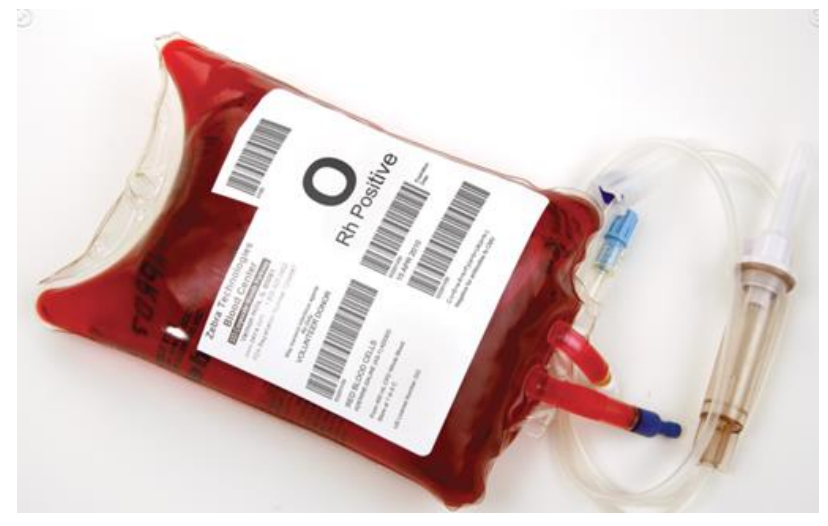

Fig. 2. blood bag marked with a barcode [10]

\section{Blood collection and registration using RFID technology}

We will now focus on what the blood center would look like after the introduction of RFID technology. The first change would occur in the first step - registration. Each donor would be equipped with their donor card with an RFID chip. It would record all the necessary information such as name and surname, photo of the donor, address, blood type, and information about previous donations. The donor would no longer fill in the questionnaire manually, after logging in with his card at the terminals at the entrance, which would also fulfill the role of registration. After completing the questionnaire, it would be assigned in the system to the donor card, along with the generation of a serial number for collection. Upon arrival at the register, the worker (who also has her card with an RFID chip) would read the reader and check the data on the donor. The donor is invited by the development device to the hematology laboratory to take a control sample. He proves himself to the employee with his ID card, which then verifies the donor's serial number and prepares him for blood collection. After taking a blood sample, the donor goes to the waiting room. The worker marks the tube with the collected blood with a smart label with an RFID chip, which she assigns to the donor card, together with her number. This will ensure control of who worked with the sample, when, and where. After the control examination of the sample, the data are entered into the computer, all information is already prepared for the examining doctor (it is no longer necessary to transfer the donor cards to the doctor). The first section was therefore significantly simplified and more operations were performed than with the barcode version.

The second section follows, namely a check-up at the doctor. The donor is again identified by his donor ID card, with which the doctor displays all the data about the donor on the computer, including the completed questionnaire and the results of the follow-up collection. He performs a control examination of the donor and records the detected data on a computer for the donor card, including his employee ID. The donor then leaves for the waiting room, where he waits for a request for blood. Again, it is not necessary to physically transfer the donor card, everything is recorded in the system.

In the third final section, the nurse no longer marks blood bags and tubing with barcodes. Canned blood will be supplied by the manufacturer with built-in RFID tags, which will contain information about the type of bag, manufacturer, batch, etc. Upon arrival, the donor presents his ID card and the nurse then loads a blood bag whose RFID tag with a specific number is assigned in the system. to the donor card, at the same time the donor's ID number is entered into the blood can database. 
Finally, the nurse ID was added to the blood bag as well as the donor ID, from which it will be clear who performed the manipulation and when. At the end of the donor's collection, the donor leaves, and the collected blood bags are moved to the production department. Their departure from the collection room is recorded by the RFID gateway.

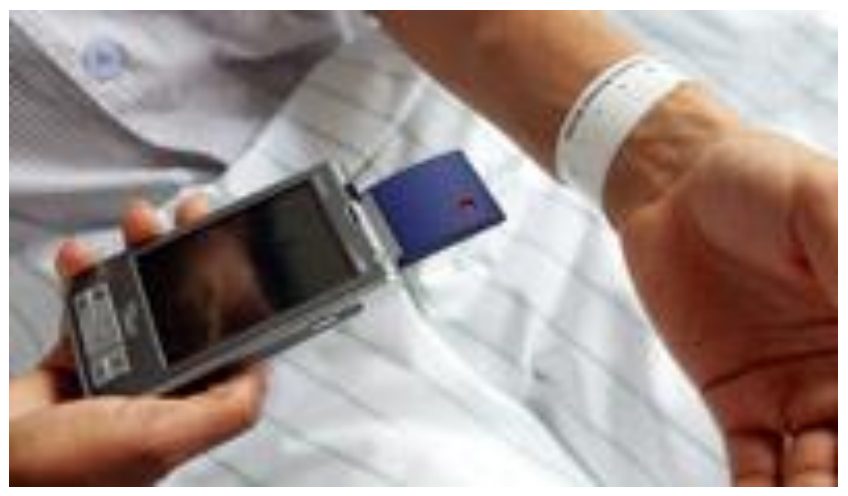

Fig. 3. The RFID chip checks the donor ID and blood cans [11]

\section{The resulting comparison and further development}

As the above comparisons clearly show, the introduction of RFID technology into the operation of any blood center would be of great benefit. The work of this department would be more accurate, clearer, and, overall, there would be a significant simplification and acceleration of individual operations. A bonus would be the maximum amount of information needed to eliminate human error. It would no longer be necessary to transfer the donor card in parallel with his movement around the ward, and the data would still be immediately available. For RFID technology to be implemented, it is necessary to create identification cards with an RFID tag for both donors and employees. Thanks to them, important data will be introduced directly into the system and tags placed on test tubes and blood bags. In the future, however, it is very important to consider which data is really necessary, because the more data is written, the slower it can be retrieved.

Individual cards and tags must contain at least the following information:

- Donor ID card: donor identification number, his name, and surname, date of birth or birth number, and address. Furthermore, blood group, RH factor, date of the last collection, and by whom it was performed, from the right or left hand it was performed, its amount, type, and whether the collection was successfully completed. If not, the reason.

- Employee ID card: employee identification number, name and surname, date of birth or birth number, department, and job position.

- RFID tag of the blood can: ID of the can, donor, employee, type of bag and bag batch, blood group, RH factor, date and type of collection, the device performing the collection.

- RFID tag of the blood sample: ID of the tube, donor and employee, blood type, RH factor, and date of collection.

It is now necessary to determine the further development of research so that the practical aspects of the introduction of this technology in medical facilities are also addressed. The design of the whole system itself must guarantee that no properties of the biological material will be damaged, especially by selecting both suitable system components and their principle of functionality. For example, for RFID tags and gateways, it is necessary to maintain the frequency of $13.56 \mathrm{MHz}$ in order not to degrade the biological material. In current practice, however, they work on UHF frequencies. Also, there may be a problem reading the tag from the blood bag or tube. Both the bag and the test tube contain a fluid for which, at the current stage of development of RFID technology, it is not possible to guarantee error-free reading of data. The same situation is with metals.

None of these reasons means the impossibility of introducing RFID technology into the operation of a blood center, because some blood centers and medical facilities in the world already use RFID technology. The introduction of this technology therefore presupposes a thorough and precise focus and verification of individual critical points, always with adaptation to the local situation and possibilities. These preparations will be not only time-consuming but also financially demanding. The argument for not financially rejecting the introduction of this technology is that expert sources state that insufficient patient identification causes $13 \%$ errors in surgical care and $67 \%$ errors in the administration of blood and blood derivatives. The identification bracelet with a chip can contribute to the identification of patients and thus to the prevention of confusion of administered drugs. It is one of the surest methods of identification, where the patient's identity cannot be verified by a query. The study showed that the use of the RFID system reduced the number of errors in the administration of drugs by $82 \%$. In this ratio, the cost of human health and life is predominant. Further research in this area will therefore continue with an accurate exploration of the possibilities of technology at the site of the specific hospital facility for which it was selected. Identification of the corresponding components of the system and its overall design, together with a financial quantification. 
Supporting arguments for the introduction of this technology will be its use in other possible systems and records, such as stock records, detection of patients and their aids, permission to enter buildings, attendance system, and others according to user needs.

\section{Acknowledgments}

This research was based on the support of the Internal Grant Agency of Tomas Bata University in Zlín, the IGA / FAI / 2020/003 project and the Institute of Security Engineering, Faculty of Applied Informatics.

\section{References}

[1] Mikeska, Karel. (2012) Application of RFID technology in the field of security (2020-09-17). Available from: https://theses.cz/id/rdwbt8/.Ostrava

[2] https://sites.google.com/site/impactofictonthelogistics/home

[3] Pokojová, Radka. (2018) Safety of patient in providing nursing care. (2020-09-17). Available from: https://theses.cz/id/5x2rob/. České Budějovice

[4] Hřib, Zdeněk and Vychytil, Pavel. (2010). A brief theoretical introduction to the issue of adverse events in the provision of health care. (2013-11-09). Available from: http://www.uzis.cz/dokumenty/strucny-teoreticky-uvod-doproblematiky-nezadoucichudalosti-pri-poskytovani-zdravotni-pece. Praha

[5] Contactless identification. (2011-12-17) <http://esp.cz/technologie/bezkontaktni-identifikace/>.

[6] Basics of RFID technology (2011-12-18)

[7] RFID technologies and systems (2011-12-17)

[8] What is RFID (2012-12-17)

[9] Rakic-Skokovic Marija, Ostojic Gordana; Lazarevic Milovan \& Stankovski Stevan. (2009). Improving Business Processes with RFID Technology, Chapter 18 in DAAAM International Scientific Book 2009, pp. 161-168, B. Katalinic (Ed.), Published by DAAAM International, ISBN 978-3-901509-69-8, ISSN 1726-9687, Vienna, Austria DOI: $10.2507 /$ daaam.scibook.2009.18

[10] http://datascan.cz/produkt/sledovani-krevnich-vaku-zebra/

[11] https://www.systemonline.cz/zpravy/rfid-cipy-hlidaji-krevni-konzervy-z.htm 